$90 \mathrm{~ms})$. There was a modest inverse correlation between DF and OI of EGMs $(R=-0.58, p<0.001)$. There was no correlation between these indices \& the same EGMs classified according to other measures (OI vs CFAEmean, $\mathrm{R}<0.01$, OI vs SCI, $\mathrm{R}=-0.11$, OI vs ContA, $\mathrm{R}=-0.06$, all $\mathrm{p}>0.5$, similar for $\mathrm{DF})$. Only $2 \pm 1 \%$ of EGMs were in the top quintile of fractionation by all 3 indices of CFEmean, Cont $\mathrm{A}$ and SCI. Only high OI (ROC AUC $=0.64 \mathrm{p}<0.01)$ at ablation site and lesion number $(\mathrm{p}<0.01)$ predicted an increase in AF cycle length. The image shows a typical left atrial map following analysis. Yellow areas represent $20 \%$ most fractionated by OI, blue represents CFAEmean, Red indicates areas of both high OI and CFAEmean. Lesions are shown, green if AFCL increased with lesion and red if AFCL was unchanged.

Conclusions The classification of fractionated EGMs is very sensitive to user-selected characteristics. Interpretation of results of "fractionation maps" must take into account analysis techniques, OI appears the most promising at guiding appropriate ablation site.

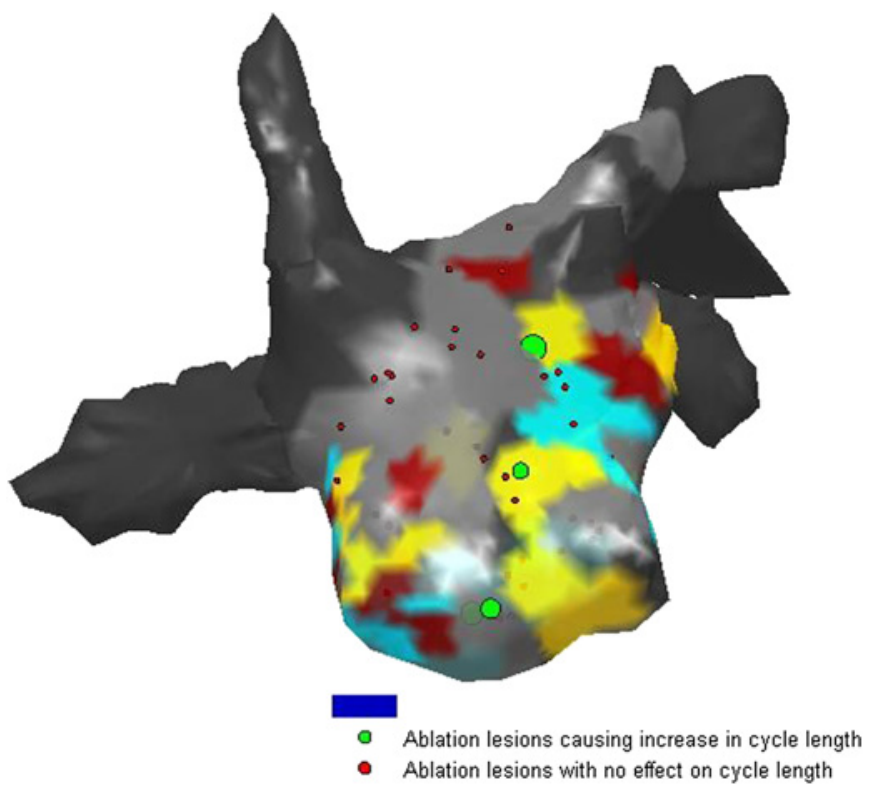

Abstract 051 Figure 1

\section{THE PREVALENCE OF LEFT ATRIAL APPENDAGE THROMBUS IN PATIENTS UNDERGOING CATHETER ABLATION FOR ATRIAL FIBRILLATION MAINTAINED ON WARFARIN}

doi:10.1136/heartjnl-2012-301877b.52

${ }^{1} \mathrm{~N}$ Herring, ${ }^{*}{ }^{2} \mathrm{~S} P$ Page, ${ }^{1} \mathrm{M}$ Ahmed, ${ }^{1} \mathrm{M}$ Burg, ${ }^{2} \mathrm{R}$ Hunter, ${ }^{2} \mathrm{M} \mathrm{J}$ Earley, ${ }^{2} \mathrm{~S} \mathrm{C}$ Sporton, ${ }^{1} \mathrm{Y}$ Bashir, ${ }^{1} \mathrm{~T}$ R Betts, ${ }^{2} \mathrm{R}$ Schilling, ${ }^{1} \mathrm{~K}$ Rajappan. ${ }^{1} \mathrm{~J} o h n$ Radcliffe Hospital, UK; ${ }^{2} \mathrm{St}$ Bartholomew's Hospital, UK

Introduction Reports of the prevalence of left atrial appendage (LAA) thrombus among patients undergoing catheter ablation for atrial fibrillation (AF) vary and may depend on the anticoagulation regime used prior to the procedure.

Methods We undertook transoesophageal echocardiograms (TOE) in 586 patients (age $59.9 \pm 0.4$ years old, mean \pm SE, $64.5 \%$ male) undergoing catheter ablation for AF who were anti-coagulated on warfarin (international normalised ratio $2-3$ ) for at least 3 consecutive weeks prior to procedure and maintained on warfarin for the procedure itself.

Results LAA thrombus was identified in 3 patients from $586(0.5 \%)$ despite all 3 having therapeutic INRs (2.2, 2.2 and 3.3 respectively). None of the remaining patients had a peri-procedural stroke. The three patients with LAA thrombus had $\mathrm{CHADS}_{2}$ scores of $\geq 1$ and CHA2DS2-VASc scores of $\geq 2$. All three patients had impaired left ventricular systolic function (LVSF), and LAA emptying velocities of $<40 \mathrm{~cm} / \mathrm{s}(23,29$ and $31 \mathrm{~cm} / \mathrm{s})$. Patients with LAA emptying velocities $<40 \mathrm{~cm} / \mathrm{s}$ on TOE $(n=111)$ had significantly $(\mathrm{p}<0.05)$ higher $\mathrm{CHADS}_{2}(0.9 \pm 0.1$ vs $0.7 \pm 0.001)$ and CHA2DS2-VASc scores $(1.7 \pm 0.1$ vs $1.4 \pm 0.1)$, and larger LA diameter $(4.95 \pm 0.09$ vs $4.38 \pm 0.05 \mathrm{~cm}$, OR for LA $>4.6 \mathrm{~cm}: 2.4,95 \%$ CI 2.13 to 5.41$)$, and were more likely to have impaired LVSF (OR: 2.66, 95\% CI 1.52 to 4.66) compared to those with higher velocities on multivariate analysis.

Conclusions The prevalence of LAA thrombus using our anticoagulation regime is extremely low. Providing patients have been therapeutically anti-coagulated, pre-operative TOE need only be performed in patients with a $\mathrm{CHADS}_{2}$ score of $\geq 1 / \mathrm{CHA} 2 \mathrm{DS} 2$-VASc score of $\geq 2$ or when LA diameter is $>4.6 \mathrm{~cm}$. This criteria has the highest sensitivity (84\%) for identifying LAA velocities of $<40 \mathrm{~cm} / \mathrm{s}$ as well as having a sensitivity of $100 \%$ for identifying thrombus and also would reduce the number of TOEs performed by $27.7 \%$.

\section{FACTORS AFFECTING QUALITY OF WARFARIN ANTICOAGULATION IN PATIENTS WITH ATRIAL FIBRILLATION: INSIGHTS FROM AFFIRM}

doi:10.1136/heartjnl-2012-301877b.53

${ }^{1} \mathrm{~S}$ Apostolakis, ${ }^{*} \mathrm{R}$ M Sullivan, ${ }^{2} \mathrm{~B}$ Olshansky, ${ }^{1} \mathrm{G}$ Y H Lip. ${ }^{1}$ Centre for Cardiovascular Sciences, City Hospital, University of Birmingham, Birmingham, UK; ${ }^{2}$ Division of Cardiovascular Medicine, University of lowa Hospitals and Clinics, lowa, USA

Introduction The efficacy of warfarin anticoagulation in atrial fibrillation patients at risk for stroke is related to time in therapeutic range (TTR) with an INR 2.0-3.0. Factors predisposing to low TTR have not been investigated comprehensively.

Methods This post hoc analysis of the AFFIRM trial included patients with at least five INR values. "Optimal" anticoagulation was defined as TTR $\geq 75 \%$; above this level, adjusted-dose warfarin offers the same prognostic benefits as new oral anticoagulants. Binary regression analysis identified independent variables associated with TTR. The impact of TTR on outcomes was assessed further through cox regression analysis.

Results Of 3066 AFFIRM patients, the mean TTR was 0.62 SD 0.2 975 patients (32\%) were "optimally" anticoagulated. These subjects were more frequently male, treated with rate control alone and were less likely to have heart failure, diabetes, myocardial infarction, and hepatic or renal failure (all $\mathrm{p}<0.05$ ). Cox regression analysis demonstrated TTR was a major determinant of all cause mortality $(p<0.001)$, ischaemic stroke or TIA $(p=0.003)$ and major bleeding $(p=0.01)$. Binary regression analysis revealed female gender $(p=0.005)$, minority status $(p<0.001)$, history of myocardial infarction $(p=0.02)$ and non-treatment with $\beta$ blockers $(p<0.001)$ were associated independently with sub-optimal anticoagulation.

Conclusion TTR is related strongly to clinical outcomes. TTR is associated with clinical and demographic characteristics. Knowledge of factors associated with low TTR may help better optimise antithrombotic management.

\section{LEFT VENTRICULAR GEOMETRY AND OUTCOME IN PATIENTS WITH ATRIAL FIBRILLATION: INSIGHTS FROM THE AFFIRM TRIAL}

doi:10.1136/heartjnl-2012-301877b.54

${ }^{1} \mathrm{~S}$ Apostolakis, ${ }^{*} \mathrm{R}$ M Sullivan, ${ }^{2} \mathrm{~B}$ Olshansky, ${ }^{1} \mathrm{G}$ Y H Lip. ${ }^{1}$ Centre for Cardiovascular Sciences, City Hospital, University of Birmingham, Birmingham, UK; ${ }^{2}$ Division of Cardiovascular Medicine, University of lowa Hospitals and Clinics, lowa, USA

Background Echo determined left ventricular (LV) hypertrophy, an established marker of cardiovascular disease, is related to prognosis 